\title{
Induction of apoptosis by an ethanol extract of Poria cocos Wolf. in human leukemia U937 cells
}

\author{
YUNG HYUN CHOI ${ }^{1,2}$ \\ ${ }^{1}$ Department of Biochemistry, Dongeui University College of Korean Medicine, Busan 614-052; \\ ${ }^{2}$ Blue-Bio Industry RIC and Anti-Aging Research Center, Dongeui University, Busan 614-714, Republic of Korea
}

Received June 14, 2015; Accepted July 31, 2015

DOI: $10.3892 / o r .2015 .4256$

\begin{abstract}
Poria cocos Wolf., which belongs to the Polyporaceae family, has been widely used as an Oriental traditional herbal medicine for centuries. Its sclerotium has been reported to possess a wide spectrum of pharmacological activities, including free-radical scavenging, anti-viral, anti-microbial, anti-inflammatory and anticancer activities. However, the cellular and molecular mechanisms of apoptosis induction by $P$. cocos in human cancer cells are poorly understood. In the present study, we investigated the pro-apoptotic potential of an ethanol extract of $P$. cocos sclerotium (EEPC) in human leukemia U937 cells in vitro. We found that EEPC induced anti-proliferative effects in U937 cells in a concentration- and time-dependent manner, which was due to apoptotic induction, as evident from morphological changes and flow cytometric assays. EEPC-induced apoptosis of U937 cells was associated with an increase in the Bax:Bcl-2 ratio, the release of cytochrome $c$ to the cytosol, and a decrease in the expression of an inhibitor of the apoptosis family of proteins. The events were accompanied by activation of caspase- $8,-9$ and -3 , and cleaved poly(ADP-ribose) polymerase, suggesting the involvement of both the intrinsic and extrinsic apoptotic cascades. In addition, the overexpression of $\mathrm{Bcl}-2$ caused a significant attenuation of EEPC-induced caspase activation, degradation of PARP, and the collapse of mitochondrial membrane potential, and thereby reversed EEPC-induced cell apoptosis and growth inhibition. Collectively, these data provide insights into the molecular mechanisms underlying EEPC-induced apoptosis in U937 cells, suggesting that EEPC may be a new therapeutic option for the treatment of leukemia.
\end{abstract}

\section{Introduction}

Apoptosis represents one of the main types of programmed cell death and is a tightly regulated cell suicide response

Correspondence to: Dr Yung Hyun Choi, Department of Biochemistry, Dongeui University College of Korean Medicine, San 45, Yangjung-dong, Busanjin-gu, Busan 614-052, Republic of Korea E-mail: choiyh@deu.ac.kr

Key words: Poria cocos Wolf., U937 cells, apoptosis, caspase, Bcl-2 that facilitates the correct development and homeostasis of multicellular organisms. Therefore, the susceptibility of cancer cells to apoptosis is an important determinant of chemotherapy efficacy $(1,2)$. Moreover, during the past decade, evidence suggests that many cancer chemotherapeutic agents kill cancer cells by inducing apoptosis. In mammalian cells, two major apoptosis pathways, the cell death receptormediated (extrinsic) and mitochondrial-mediated apoptotic (intrinsic) pathways, have been well-characterized. Both pathways are involved in an ordered activation of a highly conserved family of cysteine proteases called caspases, which in turn cleave cellular substrates, resulting in the morphological and biochemical changes characteristic of apoptosis $(2,3)$. The Bcl-2 family proteins also play a crucial role in the regulation of apoptotic events in both signaling pathways (4). They contain anti-apoptotic members, such as Bcl-2 and Bcl-xL, and pro-apoptotic members, such as Bax, Bak and Bid. Overexpression of anti-apoptotic proteins results in the prevention of apoptosis; however, overexpression of pro-apoptotic proteins leads to an increase in cell susceptibility to apoptotic signals $(4,5)$.

Recently, the demand for more effective and safer therapeutic agents for the chemoprevention of human cancer has increased (6). In that respect, fungi have been globally used as natural medicines and intensively investigated for their antitumor properties with extemely low toxic potential $(7,8)$. Among them, Poria cocos Wolf. is a saprophytic fungus in the Polyporaceae family that grows within the diverse species of Pinus. Originally, its sclerotium has been used as an important medicinal herb in several Asian countries, particularly China, Japan and Korea, for its sedative, diuretic, anti-depressant and tonic activities $(9,10)$. Recent studies have indicated that the extracts and components of this fungus display a variety of biological activities, such as anti-fungal and anti-bacterial (11), anti-oxidant (12,13), neuroprotective (14), anti-hypertonic (15), anti-inflammatory (16-19), anti-angiogenic $(20,21)$, immunomodulatory $(22,23)$ and anticancer effects $(24,25)$. Although several triterpenoids from $P$. cocos have been isolated and were demonstrated to have a cytotoxic effect against a number of human cancer cell types (26-29), the efficacy and mechanism of the whole extract in cancer treatment have not been systemically evaluated. In the present study, as a part of our ongoing screening program to evaluate the anticancer potential of medicinal fungi, we investigated the pro-apoptotic 
properties of an ethanol extract of $P$. cocos (EEPC) and the responsible underlying molecular mechanisms involved in the human leukemia U937 cell line in vitro. Our data indicated that EEPC exerts anti-proliferative effects on U937 cells through its ability to induce apoptotic cell death.

\section{Materials and methods}

Preparation of EEPC. The dried sclerotium of $P$. cocos was supplied by Dongeui University Oriental Hospital (Busan, Korea) and authenticated by Professor S.H. Hong, Department of Biochemistry, Dongeui University College of Korean Medicine. A voucher specimen (accession no. DEU-27) was deposited at the Natural Resource Bank of Dongeui University College of Korean Medicine. To prepare the EEPC, the dried sclerotium of $P$. cocos was ground into powder and extracted twice with 10 volumes of $80 \%$ ethanol at $85-90^{\circ} \mathrm{C}$ in a reflux condenser for $3 \mathrm{~h}$. After being filtered through a $0.2-\mu \mathrm{m}$ filter, the extract was concentrated and lyophilized by vacuum evaporation at $60^{\circ} \mathrm{C}$. The solid form of the extract was dissolved in dimethylsulfoxide (DMSO; Sigma-Aldrich, St. Louis, MO, USA) prior to the experiment.

Cell culture. The human leukemia U937 cells and Chang liver cells (an immortalized non-tumor cell line derived from normal liver tissue) were purchased from the American Type Culture Collection (ATCC; Manassas, VA, USA), and maintained at $37^{\circ} \mathrm{C}$ in a humidified environment $(95 \%$ air and $5 \%$ $\mathrm{CO}_{2}$ ), in RPMI-1640 supplemented with $10 \%$ heat-inactivated fetal bovine serum (FBS), $2 \mathrm{mM}$ glutamine, $100 \mathrm{U} / \mathrm{ml}$ penicillin and $100 \mu \mathrm{g} / \mathrm{ml}$ streptomycin (all from Gibco-BRL, Gaithersburg, MD, USA). Ectopic Bcl-2-overexpressing U937 (U937/Bcl-2) cells were generously provided by Professor T.K. Kwon (Department of Immunology, School of Medicine, Keimyung University, Daegu, Korea) and were maintained in a medium containing $0.7 \mu \mathrm{g} / \mathrm{ml}$ geneticin (G418 sulfate; Calbiochem, San Diego, CA, USA).

Cell viability and growth assay. For the cell viability assay, cells were seeded at a concentration of $1 \times 10^{5}$ cells $/ \mathrm{ml}$ and were treated with the indicated concentrations of EEPC for $24 \mathrm{~h}$ or with $90 \mu \mathrm{g} / \mathrm{ml}$ EEPC for the indicated times. After treatments, 3-(4,5-dimethyl-2-thiazolyl)-2,5-diphenyl-2H-tetrazolium bromide (MTT; Sigma-Aldrich) working solution was added to each culture plate and continuously incubated at $37^{\circ} \mathrm{C}$ for $3 \mathrm{~h}$. The culture supernatant was removed from the wells, and DMSO was added to completely dissolve the formazan crystals. The absorbance of each well was measured at a wavelength of $540 \mathrm{~nm}$ with an enzyme-linked immunosorbent assay (ELISA) plate reader (Molecular Devices, Sunnyvale, CA, USA) (30). Cell growth was assessed using the trypan blue dye exclusion assay. In brief, the cells were trypsinized, and viable cells were counted by trypan blue dye exclusion using a hemocytometer under an inverted microscope (Carl Zeiss, Jena, Germany). The morphological changes of cells incubated with or without EEPC for $24 \mathrm{~h}$ were examined under an inverted microscope.

Nuclear staining with DAPI. For 4',6-diamidino-2-phenylindole (DAPI; Sigma-Aldrich) staining, the cells were washed with phosphate-buffered saline (PBS) and fixed with 3.7\% paraformaldehyde (Sigma-Aldrich) in PBS for $10 \mathrm{~min}$ at room temperature. The fixed cells were washed with PBS and stained with $2.5 \mu \mathrm{g} / \mathrm{ml}$ DAPI solution for $10 \mathrm{~min}$ at room temperature. The cells were then washed twice with PBS and analyzed by fluorescence microscopy (Carl Zeiss).

Flow cytometric analysis. The cells were fixed in $70 \%$ ethanol overnight at $4^{\circ} \mathrm{C}$, washed in PBS and then resuspended in $1.12 \%$ sodium citrate buffer $(\mathrm{pH} 8.4)$ together with $12.5 \mu \mathrm{g}$ of RNase (DNase-free; Sigma-Aldrich). Incubation was continued at $37^{\circ} \mathrm{C}$ for $30 \mathrm{~min}$. The cellular DNA was then stained by applying a propidium iodide (PI) $(10 \mu \mathrm{g} / \mathrm{ml}$; Sigma-Aldrich) solution for $30 \mathrm{~min}$ at room temperature in the dark. The stained cells were analyzed using a FACSCalibur flow cytometer (Becton-Dickinson; San Jose, CA, USA). The level of apoptotic cells containing sub-G1 DNA content was determined as a percentage of the total number of cells (31).

Protein extraction and western blot analysis. For the preparation of total cellular protein, the cells were gently lysed with lysis buffer [ $40 \mathrm{mM}$ Tris ( $\mathrm{pH} 8.0$ ), $120 \mathrm{mM}, \mathrm{NaCl}, 0.5 \%$ NP-40, $0.1 \mathrm{mM}$ sodium orthovanadate, $2 \mu \mathrm{g} / \mathrm{ml}$ aprotinin, $2 \mu \mathrm{g} / \mathrm{ml}$ leupeptin and $100 \mu \mathrm{g} / \mathrm{ml}$ phenymethylsulfonyl fluoride] for $30 \mathrm{~min}$. Supernatants were collected and protein concentrations were determined using a Bio-Rad protein assay kit (Bio-Rad, Hercules, CA, USA). In a parallel experiment, the mitochondrial and cytosolic fractions were isolated using a mitochondrial and cytosolic fractionation kit (Active Motif, Carlsbad, CA, USA) according to the manufacturer's protocol. Equal amounts of protein were separated on sodium dodecyl sulfate (SDS)-polyacrylamide gels. Separated protein was transferred to nitrocellulose membranes (Schleicher \& Schuell, Keene, NH, USA) and subsequently blocked with tris-buffered saline (10 mM of Tris- $\mathrm{Cl}, \mathrm{pH}$ 7.4) containing $0.5 \%$ Tween-20 and 5\% non-fat dry milk for $1 \mathrm{~h}$ at room temperature. The proteins were probed with primary antibodies overnight at $4^{\circ} \mathrm{C}$. After probing them with the primary antibodies, the membranes were incubated with horseradish peroxidase-conjugated anti-rabbit IgG as a secondary antibody purchased from Amersham Corporation (Arlington Heights, IL, USA). Using an enhanced chemiluminescence (ECL) detection system (Amersham Corporation), immunoreactive bands were detected and exposed to an X-ray film. Primary antibodies were purchased from Santa Cruz Biotechnology, Inc. (Santa Cruz, CA, USA), Cell Signaling Technology, Inc. (Danvers, MA, USA) and Abcam (Cambridge, UK).

In vitro caspase activity assay. The activities of the caspases were determined by colorimetric assay kits (R\&D Systems, Minneapolis, MN, USA), which utilize synthetic tetrapeptides [Asp-Glu-Val-Asp (DEVD) for caspase-3; Ile-Glu-Thr-Asp (IETD) for caspase-8; Leu-Glu-His-Asp (LEHD) for caspase-9] labeled with p-nitroaniline (pNA). Briefly, cells were lysed in the supplied lysis buffer according to the manufacturer's protocol. The supernatants were collected and incubated with the supplied reaction buffer and DEVD-pNA, IETD-pNA or LEHD-pNA as substrates at $37^{\circ} \mathrm{C}$. The reactions were measured by changes in absorbance at $405 \mathrm{~nm}$ using an ELISA plate reader (32). 

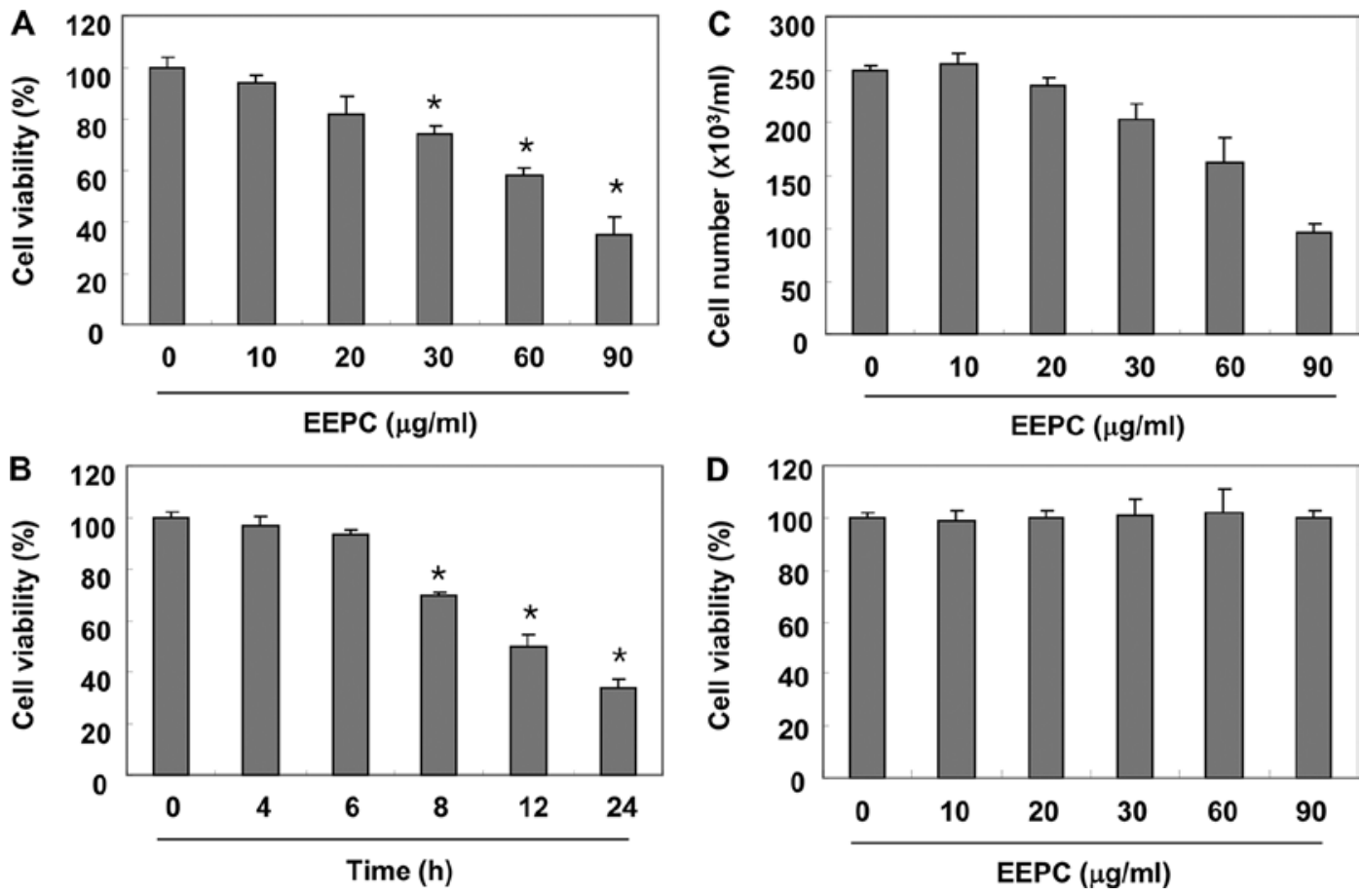

Figure 1. Effect of EEPC on cell viability in U937 cells. (A-C) U937 and (D) Chang liver cells were treated with various concentrations of EEPC for $24 \mathrm{~h}$ (A, C and D) or $90 \mu \mathrm{g} / \mathrm{ml}$ EEPC for the indicated times (B). Cell viability was measured by the metabolic-dye-based MTT assay (A, B and D), and viable cells were determined using the trypan blue dye exclusion assay $(\mathrm{C})$, respectively. Each point represents the mean \pm SD of three independent experiments. The significance was determined by Student's t-test ( $\mathrm{p}<0.05$ vs. untreated control).

Mitochondrial membrane potential (MMP) assay. The MMP of intact cells was measured by a flow cytometer using the lipophilic cationic probe 5,5',6,6'-tetrachloro-1,1',3,3'-tetraethylbenzimidazolylcarbocyanine iodide (JC-1; Calbiochem). U937 cells were collected and resuspended in PBS and then incubated with $10 \mu \mathrm{M} \mathrm{JC}-1$ for $20 \mathrm{~min}$ at $37^{\circ} \mathrm{C}$. The cells were subsequently washed once with cold PBS, suspended and subsequently analyzed using a flow cytometer (33).

Statistical analysis. All data were derived from at least three independent experiments. Statistical analyses were conducted using SigmaPlot software, and values are presented as mean \pm SD. Significant differences between the groups were determined using the unpaired Student's t-test.

\section{Results}

Induction of apoptosis in U937 cells. We first evaluated the cytotoxic effect of EEPC on U937 cells using MTT assay and trypan blue exclusion method, and found that EEPC significantly reduced the cell viability and proliferation of U937 cells in a concentration- and time-dependent manner (Fig. 1A-C). An additional experiment was conducted using Chang liver cells in order to examine the effect of EEPC on the viability of normal cells (Fig. 1D). EEPC concentrations up to $90 \mu \mathrm{g} /$ $\mathrm{ml}$ did not induce cytotoxicity. Therefore, experiments were performed to determine whether this inhibitory effect of EEPC on U937 cell growth resulted from apoptotic cell death. As shown in Fig. 2A and B, EEPC induced U937 cell death with the characteristic apoptotic features, including cell shrinkage, and chromatin condensation and fragmentation in the nucleus as detected by DAPI staining. We next quantified the apoptotic dead cells using flow cytometric analysis to detect hypodiploid cell populations. As shown in Fig. 2C, treatment of U937 cells with EEPC resulted in a markedly increased accumulation of sub-G1 phase cells, and this response occurred in a concentration-dependent manner. These results suggest an association between the growth inhibition observed in response to EEPC and the induction of apoptosis in U937 cells.

Activation of caspases by EEPC in U937 cells. We next analyzed whether treatment with EEPC results in the activation of caspases, a cardinal hallmark of apoptosis, including two initiation caspases, caspase- 8 and -9 , and the executioner caspase-3. As shown in Fig. 3A, the western blot analysis revealed that the expression levels of procaspase- $8,-9$ and -3 were all decreased or the active forms of caspase- 8 and -3 were increased in a concentration-dependent manner following EEPC treatment. In addition, quantitative determinations of caspase activities by colorimetric assays consistently showed that the activities of the three caspases were significantly increased by EEPC treatment (Fig. 3B). Furthermore, subsequent immunoblot analysis revealed that progressive proteolytic cleavage products of poly(ADPribose) polymerase (PARP), a downstream target protein of activated caspase-3 (34), occurred in U937 cells treated with EEPC. Under the same conditions, levels of the anti-apoptotic inhibitor of apoptosis proteins (IAP) family of proteins, such as XIAP, cIAP-1 and cIAP-2 (Fig. 4A), which bind to caspases and lead to their inactivation $(35,36)$, were markedly inhibited by EEPC treatment in a concentration-dependent manner. These results indicated that EEPC may trigger initiator caspase- 8 and -9 initially and subsequently activate the executioner caspase- 3 . 


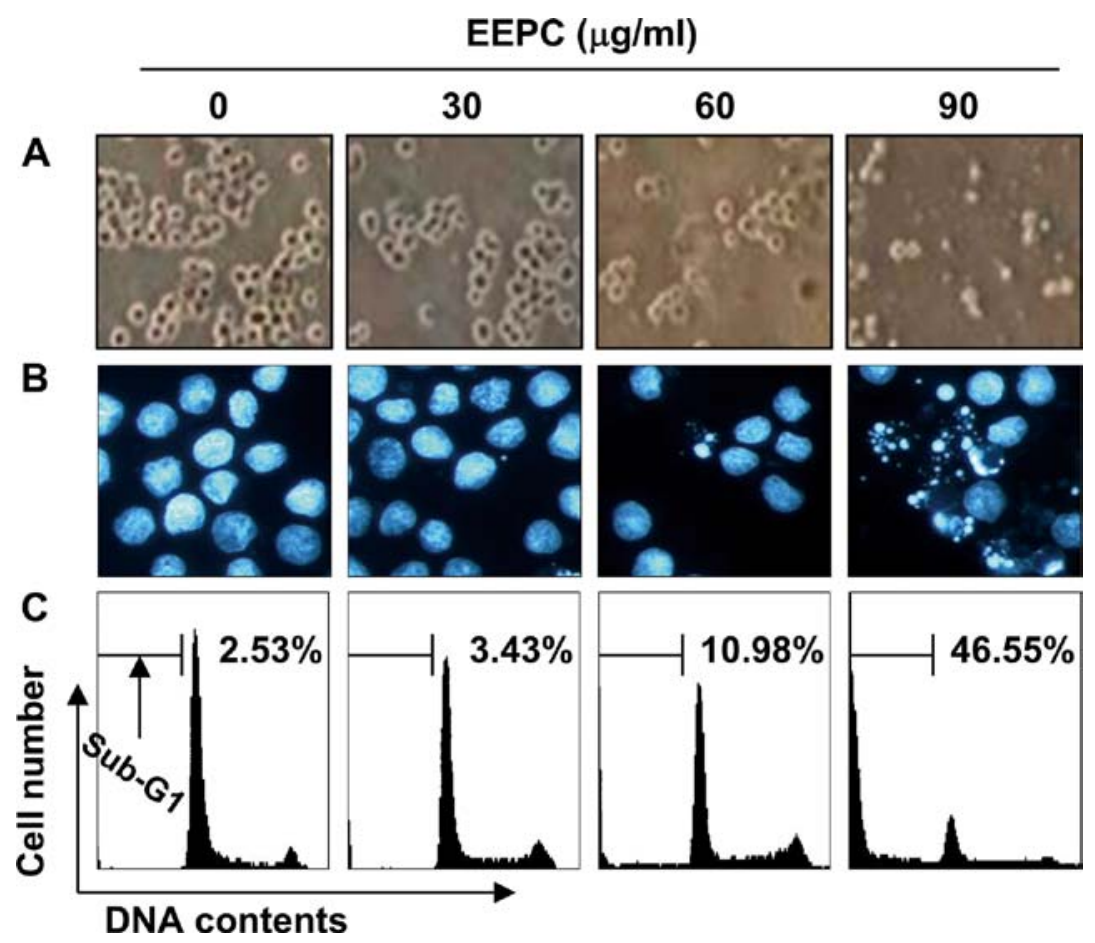

Figure 2. Induction of apoptosis by EEPC treatment in U937 cells. (A) Cells were incubated with the indicated concentrations of EEPC for $24 \mathrm{~h}$. The cellular morphology of cells was examined under a light microscope (original magnification, x200). (B) The cells were fixed and stained with DAPI solution. After a 10-min incubation at room temperature, stained nuclei were then observed using a fluorescence microscope (original magnification, $\mathrm{x} 400$ ). (C) For analyzing cell cycle distribution, the cells grown under the same conditions were harvested, and then 10,000 events were analyzed for each sample. DNA content is represented on the $\mathrm{x}$-axis and the number of cells counted is represented on the $\mathrm{y}$-axis. The data represent the average of two independent experiments.

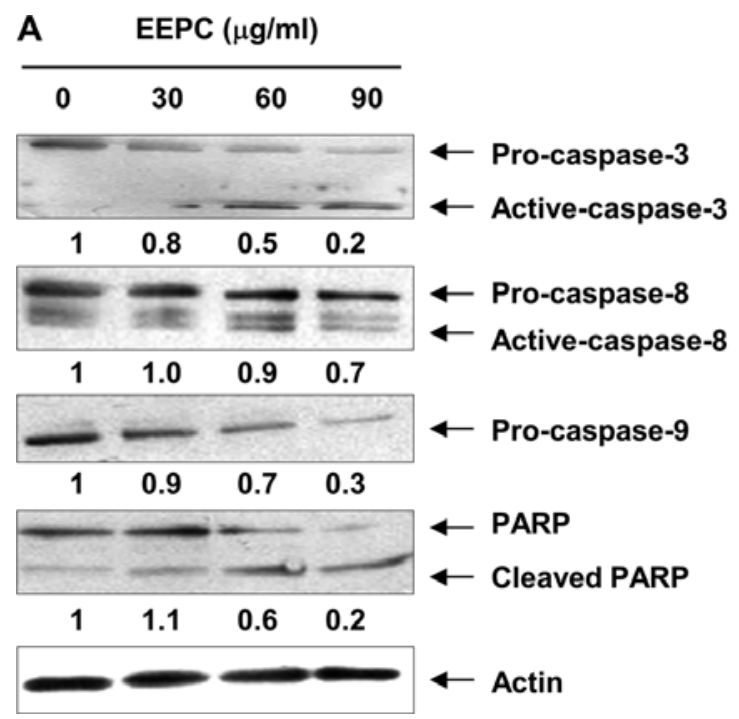

B

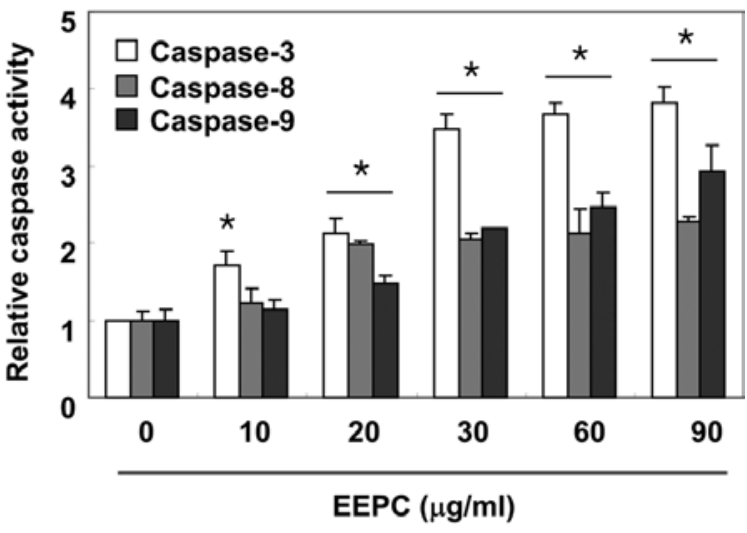

Figure 3. Activation of caspases by EEPC in U937 cells. (A) U937 cells were treated with the indicated concentrations of EEPC for $24 \mathrm{~h}$. The cells were lysed and then cellular proteins were separated by SDS-polyacrylamide gels and transferred onto nitrocellulose membranes. The membranes were probed with the indicated antibodies. Proteins were visualized using an ECL detection system. Actin was used as an internal control. The numbers represent the average densitometric analyses of pro-caspases and uncleaved PARP as compared with actin in, at a minimum, 2 or 3 different experiments. (B) Cells grown under the same conditions as (A) were lysed, and aliquots (50 $\mu \mathrm{g}$ protein) were assayed for in vitro caspase-3, -8 and -9 activity using DEVD-pNA, IETD-pNA, and LEHD-pNA as substrates, respectively, at $37^{\circ} \mathrm{C}$ for $1 \mathrm{~h}$. The released fluorescent products were measured. Data are expressed as the mean $\pm \mathrm{SD}$ of three independent experiments. The significance was determined by Student's t-test ( $\mathrm{p}<0.05$ vs. untreated control).

Modulation of Bcl-2 family members and release of cytochrome c from mitochondria to the cytosol by EEPC in U937 cells. Given that members of the Bcl-2 protein family are primarily responsible for initiating mitochondrial-mediated apoptosis, we next examined the levels of $\mathrm{Bcl}-2$ family proteins using western blot analysis. As shown in Fig. 4B, EEPC evoked a concentration-dependent reduction in the level of anti-apoptotic $\mathrm{Bcl}-2$ expression, whereas the expression level of pro-apoptotic Bax was markedly induced. The resultant alteration in Bcl-2 family protein expression apparently 

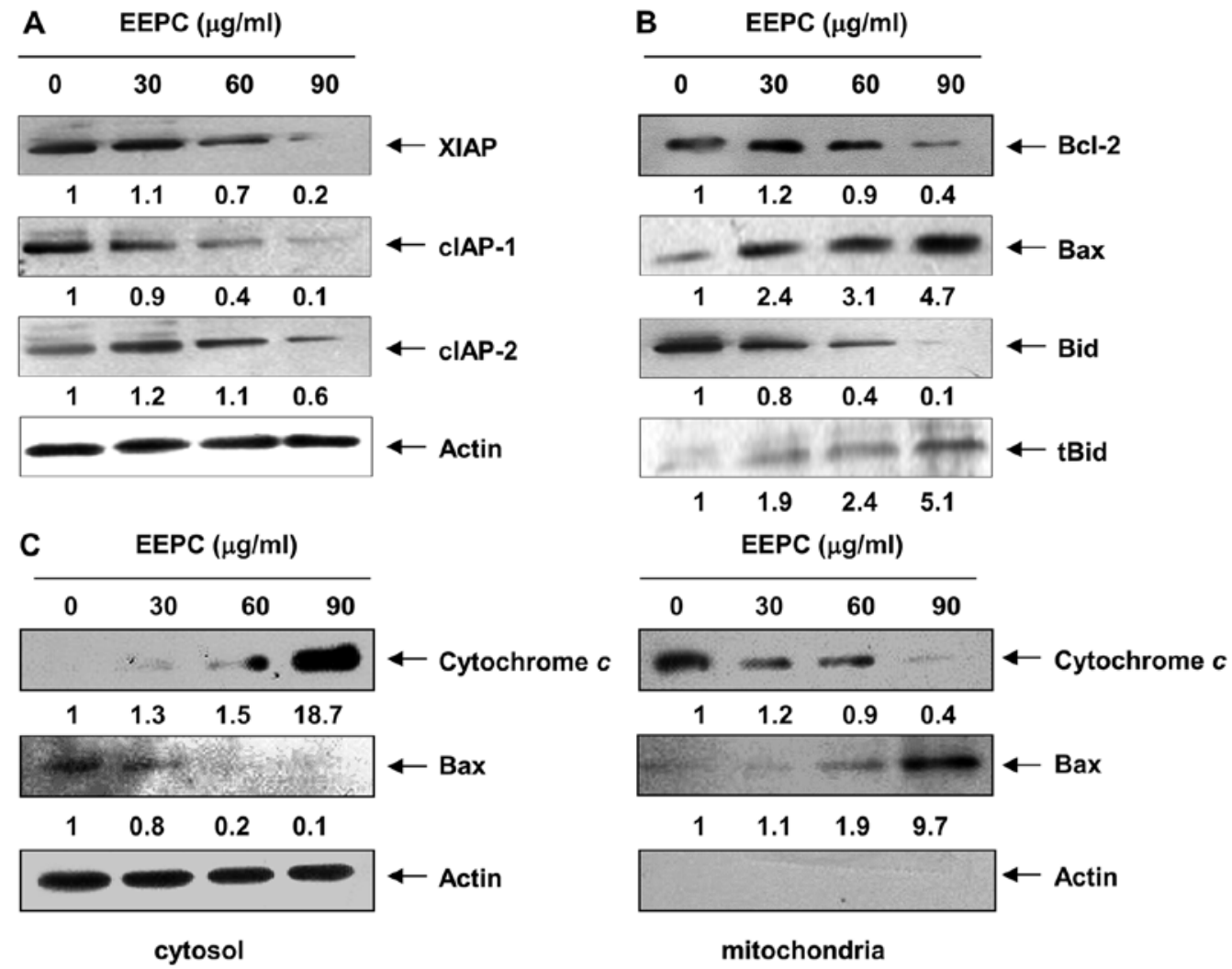

Figure 4. Modulation of IAP and Bcl-2 family proteins, and release of cytochrome $c$ to the cytosol by EEPC in U937 cells. U937 cells were treated with the indicated concentrations of EEPC for $24 \mathrm{~h}$. (A and B) The cells were lysed and then cellular proteins were separated by SDS-polyacrylamide gels and transferred onto nitrocellulose membranes. The membranes were probed with the indicated antibodies. (C) The mitochondrial and cytosolic proteins were extracted from cells grown under the same conditions and analyzed by western blotting using the indicated antibodies. Proteins were visualized using an ECL detection system. Actin was used as an internal control. The numbers represent the average densitometric analyses as compared with actin in, at a minimum, 2 or 3 different experiments.

lowered the ratio of the anti-apoptotic to the pro-apoptotic Bcl-2 family proteins, theoretically causing a disruption in mitochondrial membrane integrity and consequent cytosolic release of cytochrome $c$ to initiate caspase-9 activation $(37,38)$. Therefore, we performed an immunoblot analysis using cytosolic and mitochondrial fractions to examine the release of mitochondrial cytochrome $c$ in EEPC-treated cells and found that EEPC treatment markedly induced a dose-dependent release of cytochrome $c$ into the cytoplasm (Fig. 4C).

Translocation of Bax from the cytosol to mitochondria and Bid truncation by EEPC in U937 cells. Accumulating evidence has demonstrated that Bax protein translocation to the mitochondria is a necessary step in cell activating mitochondrial-mediated apoptosis $(39,40)$. As shown in Fig. 4C, the levels of Bax in the cytosol declined in a concentration-dependent manner after EEPC treatment. In contrast, the Bax levels in the mitochondrial fraction increased, indicating that EEPC treatment markedly led to Bax translocation into the mitochondria from the cytosol. Moreover, under the same experimental conditions, EEPC caused a concentration-dependent cleavage of $\mathrm{BH} 3$-only Bid protein and the formation of its truncated form of Bid, tBid (Fig. 4B), which could translocate to the mitochondria to enhance the mitochondrial-mediated apoptosis pathway $(41,42)$. These results suggest that EEPC reduces the Bcl-2/Bax ratio, inserts Bax from the cytosol into mitochondria, and activates Bid, resulting in mitochondrial dysfunction, release of cytochrome $c$ to the cytosol and apoptosis induction.

Effects of Bck-2 overexpression in EEPC-induced U937 cell apoptosis. Since it has been well established that Bcl-2 plays a critical role in the regulation of the mitochondrial-mediated apoptotic pathway, U937 cells stably overexpressing Bcl-2 were established and we determined the effect of overexpression of Bcl-2 on EEPC-induced apoptosis. As shown in Fig. 5A, western blot analysis revealed that Bcl-2 overexpression inhibited the EEPC-induced cleavage of caspase-3, -9 and PARP. In addition, the role of mitochondria in EEPC-induced apoptosis was further investigated by examining the effect of EEPC on MMP levels. As shown in Fig. 5B, Bcl-2 overexpression was found to significantly block cells from EEPC-induced MMP level reduction when compared to the U937/vector cells. Furthermore, Bcl-2 overexpression was found to significantly protect cells from EEPC-induced morphological changes, accumulation of cells in the sub-G1 phase and growth inhibition (Fig. 6) when compared to U937/vector cells. Taken together, these results indicated that the ectopic expression of Bcl-2 inhibits EEPC-induced apoptosis.

\section{Discussion}

As the induction of apoptosis is well recognized as the primary cytotoxic mechanism for the anticancer effect of 

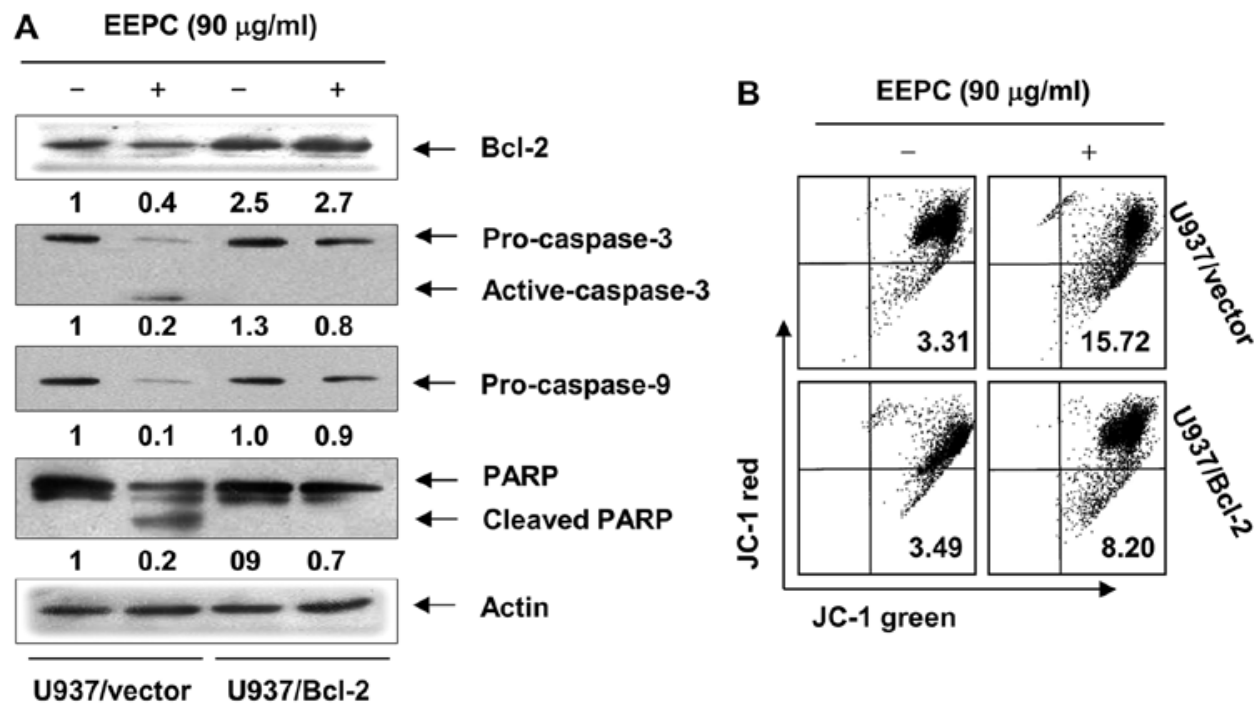

Figure 5. Effects of Bcl-2 overexpression on EEPC-induced cleavage of caspases and PARP, and loss of MMP in U937 cells. (A) Control (U937/vector) or Bcl-2transfected (U937/Bcl-2) cells were incubated with $90 \mu \mathrm{g} / \mathrm{ml}$ EEPC for $24 \mathrm{~h}$. Equal amounts of cell lysate were extracted, resolved on SDS-polyacrylamide gels, transferred to nitrocellulose membranes, and probed with anti-Bcl-2, anti-caspase-3, anti-caspase-9 and anti-PARP antibodies. Actin was used as an internal control. The numbers represent the average densitometric analyses as compared with actin in, at a minimum, 2 or 3 different experiments. (B) The MMP of cells treated under the same conditions was evaluated using a flow cytometer. The data represent the average of two independent experiments.

A
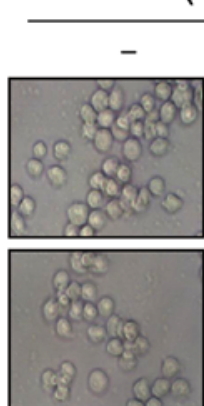

C $\operatorname{EEPC}(90 \mu \mathrm{g} / \mathrm{ml})$

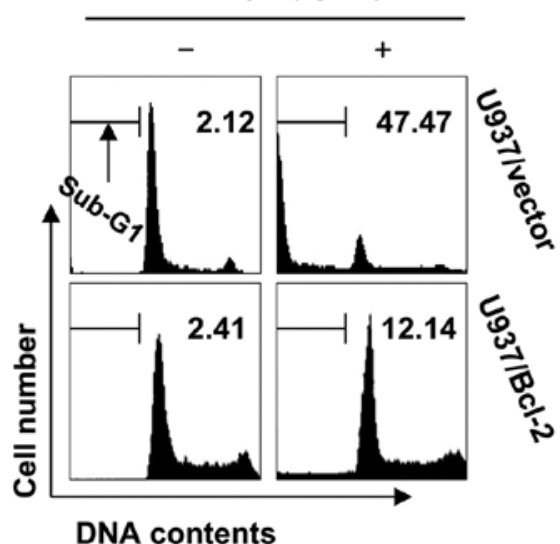

B

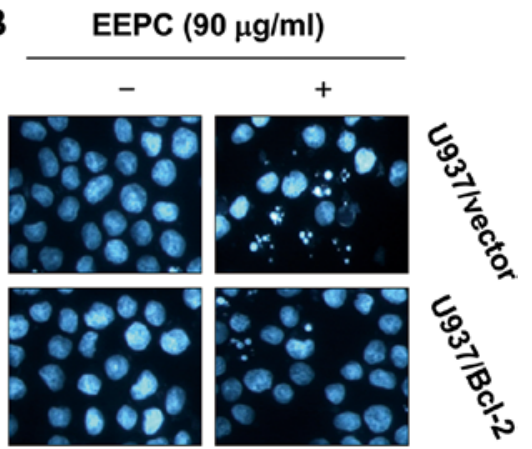

D
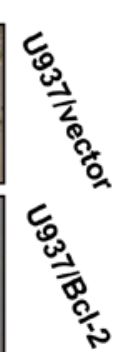

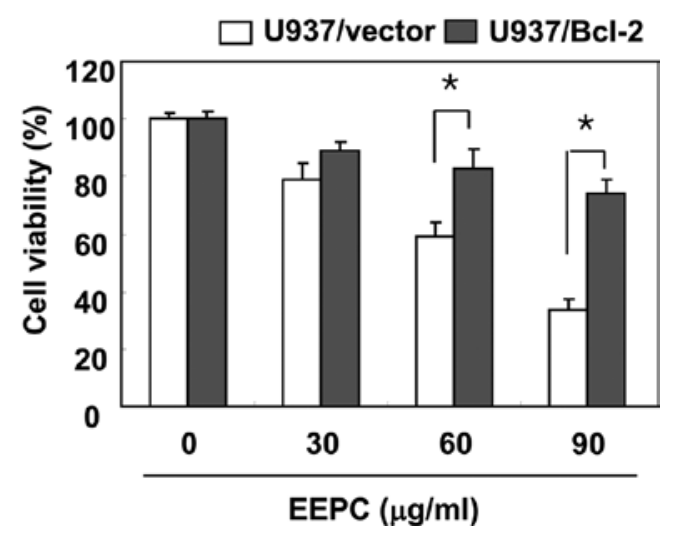

Figure 6. Effects of Bcl-2 overexpression on EEPC-induced apoptosis in U937 cells. Control (U937/vector) or Bcl-2-transfected (U937/Bcl-2) cells were incubated with $90 \mu \mathrm{g} / \mathrm{ml}$ EEPC for $24 \mathrm{~h}$. (A) The cellular morphology of cells was examined under a light microscope (original magnification, x200). (B) The cells were fixed and stained with DAPI solution. After a 10-min incubation at room temperature, stained nuclei were then observed using a fluorescence microscope (original magnification, x400). (C) The cells grown under the same conditions were collected, fixed and stained with PI for flow cytometric analysis. The percentages of cells in each cell cycle phase are presented. The data represent the average of two independent experiments. (D) Cell viability was determined by an MTT assay. Data are expressed as the mean \pm SD of three independent experiments. The significance was determined by Student's t-test ("p $<0.05$ vs. U937/vector cells).

most chemotherapeutic agents, the pro-apoptotic effect and its underlying mechanisms of EEPC were herein investi- gated. Our results demonstrated that EEPC treatment led to the activation of both initiator caspase- 8 and -9 and effector 
caspase-3, and the subsequent cleavage of PARP, in addition to the modulation of Bcl-2 and IAP family proteins. Furthermore, we found that EEPC induced the truncation of BH3-only Bid protein, translocation of Bax to the mitochondria, disruption of mitochondrial function with the loss of MMP and the release of cytochrome $c$ into the cytosol. On the other hand, Bcl-2 overexpression significantly abolished EEPC-induced cell death.

Caspases, which are a family of cysteine acid proteases, exist as pro-enzymes in the cytosol of cells and are the central regulators for the execution of cell death in response to various apoptotic stimuli $(43,44)$. Among two main apoptotic pathways, the death receptor pathway involves the engagement of a set of ligands and their corresponding receptors and then transmission of the apoptotic signal in the cytoplasm by a number of caspases such as caspase- 8 , forming the death-inducing signaling complex (3). This recruitment leads to the downstream activation of executioner caspase- 3 and -7 , and induction of apoptosis (45). Caspase-8 is involved in the activation of the mitochondrial pathway via the truncation of Bid, a BH3 domain containing a pro-apoptotic Bcl-2 family member $(41,42)$. The mitochondrial pathway can be activated by various stimuli, such as DNA damage and many types of chemotherapeutic agents $(3,46)$. In this pathway, the mitochondrialintegrity is disrupted as a result of the loss of MMP, leading to the release of cytochrome $c$ from the mitochondria to the cytosol where it can associate with apoptotic peptidase activating factor 1 (Apaf1) and pro-caspase-9, leading to the activation of caspase- 9 (37,38). Activated caspase- 9 directly cleaves and activates the executioner caspases, which leads to the cleavage of several target proteins including PARP, chromatin condensation, DNA laddering and the formation of apoptotic bodies (34). Our data showed that EEPC promoted the activation of caspase- 8 and -3 , and concomitant PARP cleavage in U937 cells (Fig. 3). We also found the activation of caspase-9, an initiator caspase of the intrinsic pathway, and a sharp decline in Bid-complete levels, together with a rise in tBid in the EEPC-treated U937 cells (Fig. 4B). Therefore, EEPC appears to induce apoptosis primarily via caspase activation-mediated intrinsic and extrinsic apoptotic pathways in U937 cells. Activation of caspases may also be regulated by a variety of proteins, including members of the IAP family, which promote cell survival after a wide variety of apoptotic stimuli elicited via intrinsic as well as extrinsic pathways through selectively binding with caspases, and as a result, inhibit caspase activity and apoptosis $(35,36)$. Our results revealed that EEPC treatment decreased the expression level of IAP family proteins (Fig. 4A), indicating that the downregulation of IAPs may be involved in the activation of caspases in EEPC-induced apoptosis in U937 cells.

The mitochondrial membrane integrity is tightly regulated by members of the Bcl-2 protein family, such as anti-apoptotic Bcl-2 and Bcl-xL, in addition to pro-apoptotic Bax and Bak $(4,47)$. Overexpression of anti-apoptotic Bcl-2 members results in the prevention of apoptosis; however, overexpression of pro-apoptotic Bcl-2 members leads to an increase in cell susceptibility to apoptotic signals $(37,47)$. In particular, a decrease in the ratio of anti-apoptotic to pro-apoptotic Bcl-2 family proteins leads to a disruption in the mitochondrial outer membrane and the consequent cytosolic release of cyto- chrome $c$ for caspase-9 activation (37,38). Moreover, it has been suggested that following initiation of the apoptotic cascade, Bax translocates from the cytoplasm to the mitochondria, and both the Bax and Bak proteins change their conformation and form homo-oligomers $(5,40)$. On the outer membrane of the mitochondria, they may form a channel or membrane pore, thus allowing the release of cytochrome $c(46,48)$. In addition to the activation of caspases, EEPC treatment resulted in a significant increase in Bax expression and a decrease in Bcl-2 expression (Fig. 4B). Accompanying modulation of Bcl-2 family proteins, Bax translocation by EEPC could be related to the mitochondrial response to the generation of tBid, leading to mitochondrial disturbance and releasing cytochrome $c$, and ultimately activating caspase- 9 to intensify the initial apoptotic response. Taken together, these findings indicate that a mitochondrial amplification step is required for complete activation of the effector caspases and apoptosis induction by EEPC in U937 cells to occur.

We further investigated the finding that Bcl-2 overexpression confers protection against EEPC-induced apoptosis in U937 cells, and Bcl-2 overexpression was found to block the EEPC-induced cleavage of caspase-3 and -9 , as well as the cleavage of PARP, a caspase-3 substrate (Fig. 5A). Additionally, Bcl-2 overexpression blocked the EEPC-induced loss of MMP in U937 cells, which indicates that Bcl-2 has a regulatory function upstream of the mitochondria (Fig. 5B). Furthermore, Bcl-2 overexpression was found to significantly protect cells from EEPC-induced apoptosis and growth inhibition, compared to U937/vector cells (Fig. 6), indicating that Bcl-2 plays a critical role in the EEPC-induced apoptosis in U937 cells.

In summary, our results demonstrated that EEPC-induced apoptosis in human leukemia U937 cells is associated with the activation of caspases at the initiative and executive stages via mitochondrial-mediated and extrinsic pathways. The results of the present study demonstrated that EEPC treatment requires a mitochondrial amplification step to enable the full activation of the caspase cascade and apoptosis induction in U937 cells to occur. In addition, our results demonstrated that $\mathrm{Bcl}-2$ overexpression inhibits EEPC-induced apoptosis by exerting effects at the mitochondrial level, as well as downstream to the mitochondria. Although additional in vivo studies are needed to establish the role of EEPC as a chemopreventive and/or therapeutic agent for leukemia and other cancers, these findings provide further elucidation of the mechanisms involved in EEPC-induced apoptosis.

\section{Acknowledgements}

The present study was supported by the Basic Science Research Program through the National Research Foundation of Korea (NRF) grant funded by the Korea government (2015R1A2A2A01004633).

\section{References}

1. Lavrik IN: Systems biology of apoptosis signaling networks. Curr Opin Biotechnol 21: 551-555, 2010.

2. Hassan M, Watari H, AbuAlmaaty A, Ohba Y and Sakuragi N: Apoptosis and molecular targeting therapy in cancer. Biomed Res Int 2014: 150845, 2014. 
3. Fulda S and Debatin KM: Extrinsic versus intrinsic apoptosis pathways in anticancer chemotherapy. Oncogene 25: 4798-4811, 2006.

4. Ola MS, Nawaz M and Ahsan H: Role of Bcl-2 family proteins and caspases in the regulation of apoptosis. Mol Cell Biochem 351: 41-58, 2011.

5. Karbowski M, Norris KL, Cleland MM, Jeong SY and Youle RJ: Role of Bax and Bak in mitochondrial morphogenesis. Nature 443: 658-662, 2006.

6. Khuda-Bukhsh AR, Das S and Saha SK: Molecular approaches toward targeted cancer prevention with some food plants and their products: Inflammatory and other signal pathways. Nutr Cancer 66: 194-205, 2014.

7. Bultman SJ: Emerging roles of the microbiome in cancer. Carcinogenesis 35: 249-255, 2014.

8. Song FQ, Liu Y, Kong XS, Chang W and Song G: Progress on understanding the anticancer mechanisms of medicinal mushroom: Inonotus obliquus. Asian Pac J Cancer Prev 14: 1571-1578, 2013.

9. Ríos JL: Chemical constituents and pharmacological properties of Poria cocos. Planta Med 77: 681-691, 2011.

10. Sun Y: Biological activities and potential health benefits of polysaccharides from Poria cocos and their derivatives. Int J Biol Macromol 68: 131-134, 2014.

11. Zhang L, Ravipati AS, Koyyalamudi SR, Jeong SC, Reddy N, Bartlett J, Smith PT, de la Cruz M, Monteiro MC, Melguizo A, et al: Anti-fungal and anti-bacterial activities of ethanol extracts of selected traditional Chinese medicinal herbs. Asian Pac J Trop Med 6: 673-681, 2013.

12. Park YH, Son IH, Kim B, Lyu YS, Moon HI and Kang HW: Poria cocos water extract (PCW) protects PC12 neuronal cells from beta-amyloid-induced cell death through antioxidant and antiapoptotic functions. Pharmazie 64: 760-764, 2009.

13. Zhou L, Zhang Y, Gapter LA, Ling H, Agarwal R and Ng KY: Cytotoxic and anti-oxidant activities of lanostane-type triterpenes isolated from Poria cocos. Chem Pharm Bull 56: 1459-1462, 2008.

14. Chung TW, Koo BS, Choi EG, Kim MG, Lee IS and Kim CH: Neuroprotective effect of a chuk-me-sun-dan on neurons from ischemic damage and neuronal cell toxicity. Neurochem Res 31: $1-9,2006$.

15. Lee SM, Lee YJ, Yoon JJ, Kang DG and Lee HS: Effect of Poria cocos on hypertonic stress-induced water channel expression and apoptosis in renal collecting duct cells. J Ethnopharmacol 141: 368-376, 2012.

16. Cuellar MJ, Giner RM, Recio MC, Just MJ, Mañez S and Rios JL: Effect of the basidiomycete Poria cocos on experimental dermatitis and other inflammatory conditions. Chem Pharm Bull 45: 492-494, 1997

17. Fuchs SM, Heinemann C, Schliemann-Willers S, Härtl H, Fluhr JW and Elsner P: Assessment of anti-inflammatory activity of Poria cocos in sodium lauryl sulphate-induced irritant contact dermatitis. Skin Res Technol 12: 223-227, 2006.

18. Jeong JW, Lee HH, Han MH, Kim GY, Hong SH, Park C and Choi YH: Ethanol extract of Poria cocos reduces the production of inflammatory mediators by suppressing the NF-kappaB signaling pathway in lipopolysaccharide-stimulated RAW 264.7 macrophages. BMC Complement Altern Med 14: 101, 2014

19. Yasukawa K, Kaminaga T, Kitanaka S, Tai T, Nunoura $Y$, Natori $\mathrm{S}$ and Takido $\mathrm{M}$ : $3 \beta$ - $p$-hydroxybenzoyldehydrotumul osic acid from Poria cocos, and its anti-inflammatory effect. Phytochemistry 48: 1357-1360, 1998.

20. Yance DR Jr and Sagar SM: Targeting angiogenesis with integrative cancer therapies. Integr Cancer Ther 5: 9-29, 2006

21. Sagar SM, Yance D and Wong RK: Natural health products that inhibit angiogenesis: A potential source for investigational new agents to treat cancer-Part 1. Curr Oncol 13: 14-26, 2006.

22. Lu YT, Kuan YC, Chang HH and Sheu F: Molecular cloning of a Poria cocos protein that activates Th1 immune response and allays $\mathrm{Th} 2$ cytokine and $\mathrm{IgE}$ production in a murine atopic dermatitis model. J Agric Food Chem 62: 2861-2871, 2014

23. Chang $\mathrm{HH}$, Yeh $\mathrm{CH}$ and Sheu F: A novel immunomodulatory protein from Poria cocos induces Toll-like receptor 4-dependent activation within mouse peritoneal macrophages. J Agric Food Chem 57: 6129-6139, 2009.

24. Cheng S, Eliaz I, Lin J, Thyagarajan-Sahu A and Sliva D: Triterpenes from Poria cocos suppress growth and invasiveness of pancreatic cancer cells through the downregulation of MMP-7. Int J Oncol 42: 1869-1874, 2013.
25. Kikuchi T, Uchiyama E, Ukiya M, Tabata K, Kimura Y, Suzuki T and Akihisa T: Cytotoxic and apoptosis-inducing activities of triterpene acids from Poria cocos. J Nat Prod 74: 137-144, 2011.

26. Feng YL, Lei P, Tian T, Yin L, Chen DQ, Chen H, Mei Q, Zhao YY and Lin RC: Diuretic activity of some fractions of the epidermis of Poria cocos. J Ethnopharmacol 150: 1114-1118, 2013.

27. Ling H, Zhang Y, Ng KY and Chew EH: Pachymic acid impairs breast cancer cell invasion by suppressing nuclear factor- $\kappa \mathrm{B}$ dependent matrix metalloproteinase- 9 expression. Breast Cancer Res Treat 126: 609-620, 2011.

28. Ling H, Zhou L, Jia X, Gapter LA, Agarwal R and Ng KY: Polyporenic acid $\mathrm{C}$ induces caspase-8-mediated apoptosis in human lung cancer A549 cells. Mol Carcinog 48: 498-507, 2009.

29. Gapter L, Wang Z, Glinski J and Ng KY: Induction of apoptosis in prostate cancer cells by pachymic acid from Poria cocos. Biochem Biophys Res Commun 332: 1153-1161, 2005.

30. Lee SJ, Hwang SO, Noh EJ, Kim DU, Nam M, Kim JH, Nam JH and Hoe KL: Transactivation of bad by vorinostat-induced acetylated $\mathrm{p} 53$ enhances doxorubicin-induced cytotoxicity in cervical cancer cells. Exp Mol Med 46: e76, 2014.

31. Kim YS, Li XF, Kang KH, Ryu B and Kim SK: Stigmasterol isolated from marine microalgae Navicula incerta induces apoptosis in human hepatoma HepG2 cells. BMB Rep 47: 433-438, 2014.

32. Park C, Park S, Chung YH, Kim GY, Choi YW, Kim BW and Choi YH: Induction of apoptosis by a hexane extract of aged black garlic in the human leukemic U937 cells. Nutr Res Pract 8: 132-137, 2014.

33. Seo K, Ki SH and Shin SM: Methylglyoxal induces mitochondrial dysfunction and cell death in liver. Toxicol Res 30: 193-198, 2014.

34. Duriez PJ and Shah GM: Cleavage of poly(ADP-ribose) polymerase: A sensitive parameter to study cell death. Biochem Cell Biol 75: 337-349, 1997.

35. Danson S, Dean E, Dive C and Ranson M: IAPs as a target for anticancer therapy. Curr Cancer Drug Targets 7: 785-794, 2007.

36. de Graaf AO, de Witte T and Jansen JH: Inhibitor of apoptosis proteins: New therapeutic targets in hematological cancer? Leukemia 18: 1751-1759, 2004

37. Kadenbach B, Arnold S, Lee I and Hüttemann M: The possible role of cytochrome $c$ oxidase in stress-induced apoptosis and degenerative diseases. Biochim Biophys Acta 1655: 400-408, 2004.

38. Scorrano L and Korsmeyer SJ: Mechanisms of cytochrome $c$ release by proapoptotic BCL-2 family members. Biochem Biophys Res Commun 304: 437-444, 2003.

39. Stankiewicz AR, Lachapelle G, Foo CP, Radicioni SM and Mosser DD: Hsp70 inhibits heat-induced apoptosis upstream of mitochondria by preventing Bax translocation. J Biol Chem 280: 38729-38739, 2005.

40. Degli Esposti M and Dive C: Mitochondrial membrane permeabilisation by Bax/Bak. Biochem Biophys Res Commun 304: 455-461, 2003.

41. Shamas-Din A, Brahmbhatt H, Leber B and Andrews DW: BH3-only proteins: Orchestrators of apoptosis. Biochim Biophys Acta 1813: 508-520, 2011

42. Fennell DA and Chacko A: Exploiting BH3 only protein function for effective cancer therapy. Front Biosci 13: 6682-6692, 2008.

43. Fiandalo MV and Kyprianou N: Caspase control: Protagonists of cancer cell apoptosis. Exp Oncol 34: 165-175, 2012.

44. Hensley P, Mishra M and Kyprianou N: Targeting caspases in cancer therapeutics. Biol Chem 394: 831-843, 2013.

45. Walczak H and Krammer PH: The CD95 (APO-1/Fas) and the TRAIL (APO-2L) apoptosis systems. Exp Cell Res 256: 58-66, 2000.

46. Asakura T and Ohkawa K: Chemotherapeutic agents that induce mitochondrial apoptosis. Curr Cancer Drug Targets 4: 577-590, 2004.

47. Tomek M, Akiyama T and Dass CR: Role of Bcl-2 in tumour cell survival and implications for pharmacotherapy. J Pharm Pharmacol 64: 1695-1702, 2012.

48. Jourdain A and Martinou JC: Mitochondrial outer-membrane permeabilization and remodelling in apoptosis. Int $\mathrm{J}$ Biochem Cell Biol 41: 1884-1889, 2009. 